\title{
Predicting the response of the dental pulp to SARS-CoV2 infection: a transcriptome-wide effect cross-analysis
}

\author{
Johnah C. Galicia $\mathbb{( i )}^{1} \cdot$ Pietro H. Guzzi ${ }^{2}$ Federico M. Giorgi ${ }^{3}$ Asma A. Khan $\mathbb{(}^{4}$
}

Received: 10 June 2020 / Revised: 29 August 2020 / Accepted: 21 September 2020 / Published online: 3 October 2020

(c) The Author(s), under exclusive licence to Springer Nature Limited 2020

\begin{abstract}
Pulpitis, inflammation of the dental pulp, is a disease that often necessitates emergency dental care. While pulpitis is considered to be a microbial disease primarily caused by bacteria, viruses have also been implicated in its pathogenesis. Here, we determined the expression of the SARS-CoV2 receptor, angiotensin converting enzyme 2 (ACE2) and its associated cellular serine protease TPMRSS2 in the dental pulp under normal and inflamed conditions. Next, we explored the relationship between the SARS-CoV-2/human interactome and genes expressed in pulpitis. Using existing datasets we show that both ACE2 and TPMRSS2 are expressed in the dental pulp and, that their expression does not change under conditions of inflammation. Furthermore, Master Regulator Analysis of the SARS-CoV2/human interactome identified 75 relevant genes whose expression values are either up-regulated or down-regulated in both the human interactome and pulpitis. Our results suggest that the dental pulp is vulnerable to SARS-CoV2 infection and that SARS-CoV-2 infection of the dental pulp may contribute to worse outcomes of pulpitis.
\end{abstract}

\section{Introduction}

SARS-CoV2 is a highly aggressive coronavirus, which has infected over 6 million people to date [1]. Most infections $(81 \%)$ produce only mild symptoms or are asymptomatic. Fifteen percent of infections are severe and require hospitalization. Transmission of this disease is produced by asymptomatic carriers, symptomatic patients, as well patients who are in the incubation period. Human-to-human transmission occurs via close contact of respiratory droplets, direct contact with infected individuals, or by contact with contaminated objects and surfaces.

$\triangle$ Asma A. Khan

khana2@uthscsa.edu

1 Department of Endodontics, Arthur A. Dugoni School of Dentistry, University of the Pacific, San Francisco, CA 94103, USA

2 Department of Surgical and Medical Sciences, Magna Graecia University, Campus S. Venuta, Catanzaro 88100, Italy

3 Department of Pharmacy and Biotechnology, University of Bologna, Bologna 40126, Italy

4 Department of Endodontics, School of Dentistry, University of Texas Health and Sciences Center, San Antonio, TX 78229, USA
Viral entry into the target cells requires angiotensin converting enzyme 2 (ACE2) and its associated cellular serine protease TPMRSS2 [2]. ACE2, a negative regulator of the renin-angiotensin system, functions as the key SARS coronavirus receptor and stabilizer of neutral amino acid transporters [3]. Viruses found to use this protein for cell entry include Influenza virus and the human coronaviruses HCoV-229E, MERS-CoV, SARS-CoV, and SARS-CoV-2. It is postulated that the pattern of expression of ACE2 in human respiratory epithelia and oral mucosa explains the rapid human-human transmission [4]. TMPRSS2, a serine protease, facilitates entry of viruses into host cells by proteolytically cleaving and activating viral envelope glycoproteins. While a number of studies have examined the expression of ACE2 and TPMRSS2 in various tissues, their expression in the dental pulp is yet to be examined.

Pulpitis, inflammation of the dental pulp, is a disease that often necessitates emergency dental care. Over $90 \%$ of dental emergency visits are due to pulpitis pain [5]. In the United States alone, over 22 million procedures are performed annually to treat diseased dental pulps [6]. While pulpitis is considered to be a microbial disease primarily caused by bacteria [7], viruses have also been implicated in its pathogenesis [8]. The complex interaction between the 31 SARS-CoV2 and human proteins has been recently 
reported [9], but the correlation between this interactome and pulpitis is yet to be explored.

Here, we first used existing gene expression data $(53,000$ genes) from our previously published study on pulpitis (GEO NCBI accession number GSE77459; ID: 20007745) [10]. Then, with the data and methods used in a recently published study on the transcriptome-wide effects on coronavirus infection in human cells [11], a master regulator analysis (MRA) [12] was performed to identify genes whose expression values are correlated (upregulated or downregulated) in both the human interactome [11] and pulpitis datasets [10]. With these techniques, we endeavored to answer two important questions: how would the dental pulp respond to SARS-CoV2 infection, and how would ACE2 and TPMRSS2 expression change during SARSCoV2 infection?

\section{Results and discussion}

We identified 75 relevant genes whose expression values are either upregulated or downregulated in both human interactome and pulpitis datasets (Table 1). Of particular interest amongst these genes are ACE2 and TMPRSS2, which were shown to be exploited by SARS-CoV-2 for cell entry and for spike (S) protein priming, respectively [9].

In the human dental pulp, both ACE2 and TMPRSS2 are expressed consistently in biopsies of normal and inflamed tissues (Fig. 1a, b). The expression levels of both ACE2 and TMPRSS2 remain unchanged under conditions of inflammation. These findings are similar to expression patterns in other tissues. For example, both of these viral entry molecules are expressed in the ileum and colon and, expression levels in mucosal biopsies of these tissues do not differ between patients with active Irritable Bowel Disease and those of control patients [13]. Similarly, the expression level of ACE2 in lung tissues does not differ between biopsies taken from patients with chronic respiratory diseases such as chronic obstructive pulmonary diseases and asthma compared to those from healthy volunteers [14].

It is important to note that the consistent expression of both ACE2 and TMPRSS2 in the dental pulp confers its vulnerability to SARS-Cov2 infection. The oral cavity stands at the entry of the respiratory system where its fluids like saliva have been reported to harbor and transmit SARSCoV2 [15]. This vulnerability may also increase the risk of infection in dental personnel. While several studies have documented the risk of COVID-19 in health care workers, they have been mostly limited to those in medicine and surgery. To our knowledge the risk of acquiring SARSCoV2 or developing COVID-19 in dental personnel is yet to be documented.
Table 1 Master regulator analysis (MRA) between the gene microarray datasets of inflamed human dental pulp tissue cells and SARS-Cov2 transcriptome in human lung tissue cells.

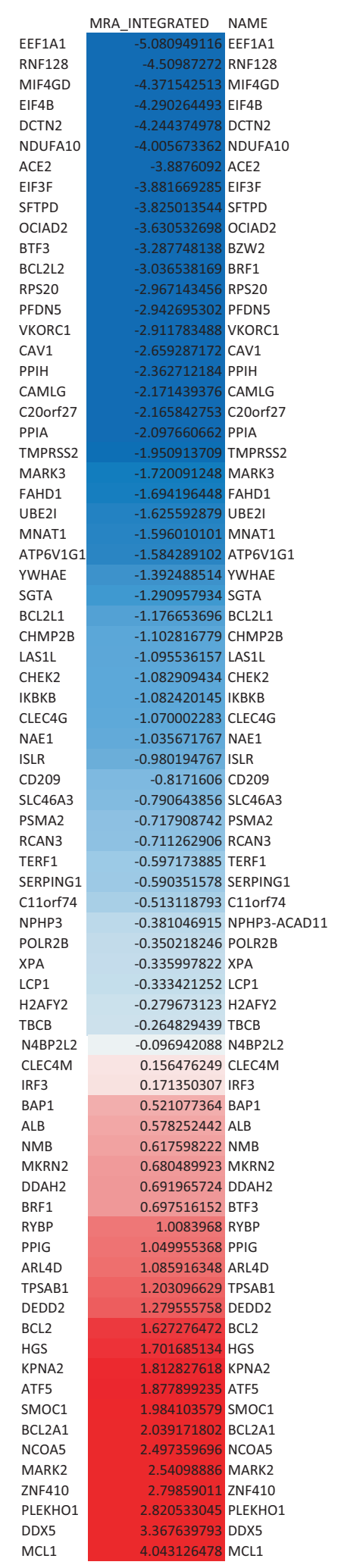

Underexpressed genes are highlighted in blue, overexpressed genes in red. 
Fig. 1 ACE2 and TMPRSS2 expression in normal and inflamed human dental pulps. Differences in RMA $\log 2$ signal intensity between samples were analyzed using Student's $t$ test. No statistical difference was found in the expression of both genes in normal and inflamed dental pulps.
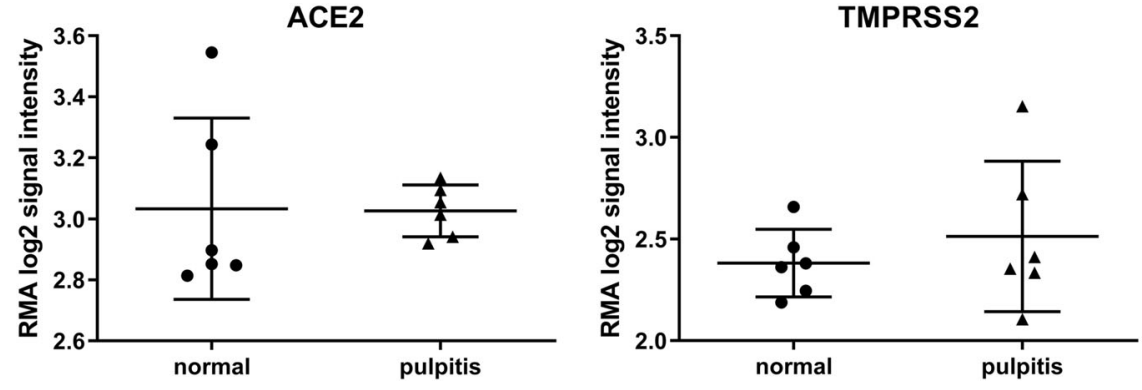

The MRA correlation analysis between the pulpitis microarray dataset and the human SARS-Cov2 transcriptome-wide effects shows that both ACE2 and TMPRSS2 values are underexpressed (Table 1). SARS$\mathrm{CoV}$ infection in other tissues have shown marked decrease in ACE2 expression [16]. Furthermore, underexpression of ACE2 was associated with worse outcomes of SARS-CoV2 infection in patients with inflammatory bowel disease [17].

One of the advantages of studies like the present one is that by using omic tools, one can answer several biological questions, without the need of collecting more samples. Our original studies on gene expression in inflamed human pulps was conducted several years ago. We then took advantage of having the sequenced data to explore expression of ACE2 and TPMRSS2 in inflamed and normal human pulps. This was an efficient and quick way to answer a time-sensitive question [10].

Taken together, our results suggest that the dental pulp is vulnerable to SARS-CoV2 infection. The predicted underexpression of ACE2 during SARS-CoV infection in the dental pulp may contribute to worse outcomes of pulpitis.

\section{Materials and methods}

Our analysis started by assessing a published study that utilized a dataset describing the transcriptome-wide effects of coronavirus infection in human cells [11]. The study employed a system [18] that probed the transcriptome-wide effects of SARS-CoV2 and its implication on human interactome by applying a MRA, which was performed by comparing infected and mock samples in both MERS and SARS datasets separately with the corto algorithm [18]. With these tools, an MRA correlation analysis of the gene microarray dataset of pulpitis [10] can be performed using a known platform in managing microarray binary data [19].

We first calculated a gene-by-gene signature of differential expression of genes caused by viral infections. In brief, a gene-by-gene signature of viral-induced differential expression is generated, and combined value for each coexpression network is generated by weighting every gene's likelihood in the network, providing a final Normalized Enrichment Score for each genes of the human/Sars-Cov2 integrated interactome. The value is positive when the network is unregulated by the infections, and vice-versa. In this study, we hypothesized that the effects of the viral infection are the same for the dental tissue, therefore we merged the data published in human airway cells [11] with that in dental pulp tissue cells [10]. Consequently, we were able to predict a possible regulator effect for the same genes. This study may therefore suggest some possible effects that should be tested in the future by extracting dental tissue samples from Covid-19 infected patients.

Gene expression analysis of ACE2 and TMPRSS2 in normal and inflamed dental pulps were from our previous study's existing public gene expression database on pulpitis (GEO NCBI accession number GSE77459). Differences in RMA $\log 2$ signal intensity between samples were analyzed using Student's $t$ test. Statistical significance was set at 0.05 .

Acknowledgements We are grateful to Dr. Xiaoyuan Han for her assistance with statistical analysis

\section{Compliance with ethical standards}

Conflict of interest The authors declare that they have no conflict of interest.

Publisher's note Springer Nature remains neutral with regard to jurisdictional claims in published maps and institutional affiliations.

\section{References}

1. WHO. World Health Organization: Coronavirus. 2019. https://www. who.int/emergencies/diseases/novel-coronavirus-2019.

2. Hoffmann M, Kleine-Weber H, Schroeder S, Kruger N, Herrler T, Erichsen S, et al. SARS-CoV-2 cell entry depends on ACE2 and TMPRSS2 and is blocked by a clinically proven protease inhibitor. Cell. 2020;181:271-80 e8.

3. Kuba K, Imai Y, Penninger JM. Multiple functions of angiotensinconverting enzyme 2 and its relevance in cardiovascular diseases. Circ J. 2013;77:301-8. 
4. Xu H, Zhong L, Deng J, Peng J, Dan H, Zeng X, et al. High expression of ACE2 receptor of 2019-nCoV on the epithelial cells of oral mucosa. Int J Oral Sci. 2020;12:8.

5. Allareddy V, Rampa S, Lee MK, Allareddy V, Nalliah RP. Hospital-based emergency department visits involving dental conditions: profile and predictors of poor outcomes and resource utilization. J Am Dent Assoc. 2014;145:331-7.

6. AAE. American Association of Enododontists: endodontics treatment statistics. https://www.aae.org/specialty/about-aae/new s-room/endodontic-treatment-statistics/.

7. Blome B, Braun A, Sobarzo V, Jepsen S. Molecular identification and quantification of bacteria from endodontic infections using real-time polymerase chain reaction. Oral Microbiol Immunol. 2008;23:384-90.

8. Li H, Chen V, Chen Y, Baumgartner JC, Machida CA. Herpesviruses in endodontic pathoses: association of Epstein-Barr virus with irreversible pulpitis and apical periodontitis. J Endod. 2009;35:23-9.

9. Srinivasan S, Cui H, Gao Z, Liu M, Lu S, Mkandawire W. et al. Structural genomics of SARS-CoV-2 indicates evolutionary conserved functional regions of viral proteins. Viruses. 2020;12:360.

10. Galicia JC, Henson BR, Parker JS, Khan AA. Gene expression profile of pulpitis. Genes Immun. 2016;17:239-43.

11. Guzzi PH, Mercatelli D, Ceraolo C, Giorgi FM. Master regulator analysis of the SARS-CoV-2/human interactome. J Clin Med. 2020;9:982.

12. Lefebvre C, Rajbhandari P, Alvarez MJ, Bandaru P, Lim WK, Sato M, et al. A human B-cell interactome identifies MYB and
FOXM1 as master regulators of proliferation in germinal centers. Mol Syst Biol. 2010;6:377.

13. Burgueno JF, Reich A, Hazime H, Quintero MA, Fernandez I, Fritsch $\mathrm{J}$, et al. Expression of SARS-CoV-2 Entry Molecules ACE2 and TMPRSS2 in the Gut of Patients With IBD. Inflamm Bowel Dis. 2020;26:797-808.

14. Li G, He X, Zhang L, Ran Q, Wang J, Xiong A. Assessing ACE2 expression patterns in lung tissues in the pathogenesis of COVID19. J Autoimmun.2020;12:102463.

15. To KK, Tsang OT, Chik-Yan Yip C, Chan KH, Wu TC, Chan JMC. et al. Consistent detection of 2019 novel coronavirus in Q2623 saliva. Clin Infect Dis. 2020;71:841-3.

16. Oudit GY, Kassiri Z, Jiang C, Liu PP, Poutanen SM, Penninger $\mathrm{JM}$, et al. SARS-coronavirus modulation of myocardial ACE2 expression and inflammation in patients with SARS. Eur J Clin Investig. 2009;39:618-25.

17. Potdar A, Dube S, Naito T, Botwin G, Haritunians T, Li D, et al. Reduced expression of COVID-19 host receptor, ACE2 is associated with small bowel inflammation, more severe disease, and response to anti-TNF therapy in Crohn's disease. 2020. https://www.medrxiv.org/content/10.1101/2020.04.19. $20070995 \mathrm{v} 1$.

18. Mercatelli D, Lopez-Garcia G, Giorgi FM. corto: a lightweight R package for gene network inference and master regulator analysis. Bioinformatics. 2020;36:3916-7.

19. Guzzi PH, Cannataro M. mu-CS: an extension of the TM4 platform to manage Affymetrix binary data. BMC Bioinform. 2010;11:315. 